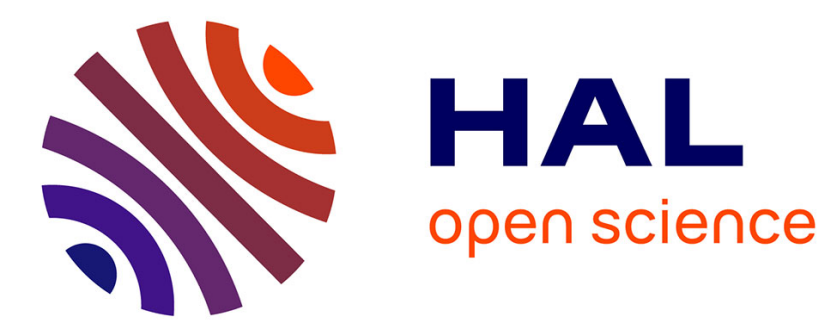

\title{
Vehicle Lateral Velocity and Lateral Tire-road Forces Estimation Based on Switched Interval Observers
}

\author{
Sara Ifqir, Dalil Ichalal, Naïma Ait-Oufroukh, Saïd Mammar
}

\section{To cite this version:}

Sara Ifqir, Dalil Ichalal, Naïma Ait-Oufroukh, Saïd Mammar. Vehicle Lateral Velocity and Lateral Tire-road Forces Estimation Based on Switched Interval Observers. The 2020 American Control Conference (ACC 2020), Jul 2020, Denver, CO, United States. pp.3023-3028, 10.23919/ACC45564.2020.9147793 . hal-02877867

\section{HAL Id: hal-02877867 https://hal.science/hal-02877867}

Submitted on 22 Jun 2020

HAL is a multi-disciplinary open access archive for the deposit and dissemination of scientific research documents, whether they are published or not. The documents may come from teaching and research institutions in France or abroad, or from public or private research centers.
L'archive ouverte pluridisciplinaire HAL, est destinée au dépôt et à la diffusion de documents scientifiques de niveau recherche, publiés ou non, émanant des établissements d'enseignement et de recherche français ou étrangers, des laboratoires publics ou privés. 


\title{
Vehicle Lateral Velocity and Lateral Tire-road Forces Estimation Based on Switched Interval Observers
}

\author{
Sara Ifqir, Dalil Ichalal, Naima Ait-Oufroukh and Saïd Mammar
}

\begin{abstract}
Lateral velocity and tire-road forces are vital signals that affect the stability of a vehicle under cornering. Unfortunately, for both technical and economic reasons, these fundamental vehicle parameters can hardly be measured directly through sensors. As a consequence, an efficient and reliable algorithm for estimating vehicle lateral velocity and tireroad forces is needed. This paper presents a novel framework for estimation of vehicle lateral velocity and lateral tire-road forces. The proposed algorithm is based on switched interval observers and is able to cope with changes of tire operating conditions. The interval estimation algorithm is evaluated through experimental data acquired using an instrumented vehicle. Simulation results show that the developed system can reliably estimate the upper and lower bounds of vehicle lateral variables during both steady and transient maneuvers.
\end{abstract}

\section{INTRODUCTION}

Nowadays, automotive embedded electronic technologies are increasingly developed to assist drivers, improve handling and maintain vehicle stability. Several active safety systems, such as Adaptive Cruise Control [24], Electronic Stability Program [9], Lane Departure Warning [23] and Lane Keeping [13] have become standard equipments in commercialized vehicles. Although these systems are considered as life-saving technologies, they are limited by the available vehicle state information. Actually, these systems rely only on the inexpensive measurements such as steering angle, yaw rate, longitudinal velocity, accelerations and so on. However, some other critical vehicle parameters, such as vehicle lateral velocity, sideslip angle and tire/road forces are difficult to measure due to either technical and economic reasons. These crucial factors could greatly improve vehicle safety, performance and comfort. Therefore, virtual sensing techniques (i.e. Estimation) are recommended.

A basic requirement for autonomous vehicle systems operating in unstructured environments is the ability to efficiently estimate the state in the presence of parameter uncertainties and disturbance inputs. Most existing methods for estimation of vehicle lateral dynamics state rely on a deterministic analysis that assumes accurate knowledge of the vehicle parameters [18], [10], [22] and [19]. However, in field conditions, vehicle parameters, such that, mass, location of the center of gravity and cornering stiffness at the front and rear tires, might have significant uncertainties due to vehicle motions, different load conditions, and road frictions. Note that, the most crucial parameters of the vehicle dynamics that represent lateral grip conditions and are difficult or

All the authors are with the Laboratory of Computing, Bio-Informatics and Complex Systems (IBISC), Univ Evry, Paris-Saclay University, Evry 91000, France Email: \{sara.ifqir, dalil.ichalal, naima.aitoufroukh, said.mammar\}@ibisc.univ-evry.fr impossible to be accurately measured are the tires cornering stiffness. These parameters vary depending on several factors such as vehicle motion and environmental conditions. For this reason, the effects of the parameter uncertainties on the estimation errors should be taken into account in order to achieve desirable level of robust performance. The approach proposed in this work to satisfactorily estimate vehicle lateral state variables, consists in assuming that the value of cornering stiffness is unknown but bounded within a priori known bounds. Then, by performing a suitable decomposition of the lateral dynamics model according to the longitudinal velocity values, a switched interval observer is constructed in order to provide the admissible sets of vehicle lateral velocity and lateral tire forces. The provided compact set is consistent with the measurement, the vehicle model and the bounded uncertainties.

Recently, interval estimation based-approach has attracted the interest of many researchers and important results are available in the literature for different class of dynamical systems [3], [4], [6], [11], [14]. They were originally developed in [5] for the estimation of biological systems subject to unknown uncertainties. Despite the abundant literature on the design of interval state observers, their applications for the development of safety-critical automotive applications problems is still missing.

The main goal of this work is to employ this guaranteed estimation method in order to accurately estimate the vehicle lateral velocity and lateral tire-road forces. This study presents significant particularities:

1) The interval estimation requires only upper and lower bounds of tire-road parameters, which can be easily identified to represent a realistic estimate of the variation range (from dry asphalt to ice-covered roads).

2) The dynamic behavior of the tire is taken into account when evaluating the generated lateral tire force, by means of relaxation tire models [17], while the most of the existing studies use a static tire model;

3) In order to demonstrate the effectiveness of the interval estimation methodology, a scenario based on experimental analysis using real data is presented.

The remainder of the paper is organized as follows. Section II discusses and describes the vehicle lateral dynamics modeling. Section III illustrates the interval observer design and formulates the condition of existences as an optimization problem in terms of Linear Matrix Inequalities (LMIs). In section IV, the simulation results are discussed and compared to real experimental data. Section V concludes the paper. 


\section{Vehicle Lateral Dynamics Modeling}

Vehicle lateral dynamics may be modeled using a two degree of freedom (2-DOF) model known as the "bicycle model" to describe the lateral translation and yaw motion [15]. The vehicle's left and right wheels are grouped together to form a single steerable front wheel and rear wheel with negligible inertia. This representation, also known as "single track model", has been proven to perfectly represent the vehicle lateral dynamics behavior. Especially, when evaluating lateral velocity angle and studying lateral efforts. The twodimensional model describing the vehicle lateral behavior can be represented by the following differential equations:

$$
\left\{\begin{array}{l}
m \dot{v}_{y}+m v_{x} \dot{\psi}=F_{y f}+F_{y r} \\
I_{z} \ddot{\psi}=l_{f} F_{y f}-l_{r} F_{y r}
\end{array}\right.
$$

where $m, I_{z}$, are the mass and the yaw moment, $v_{x}$ and $v_{y}$ are longitudinal and lateral velocities, $\dot{\psi}$ is the yaw rate, $l_{f}, l_{r}$ are distances from front and rear axle to the center of gravity (CG), while $F_{y f}$ and $F_{y r}$ are lateral tire force of front and rear tires.

The lateral front and rear forces $F_{y f}$ and $F_{y r}$ are expressed by the Pacejka's magic formula [12] as

$$
F_{y i}^{S}=D_{i} \sin \left(C_{i} \tan ^{-1}\left(B_{i}\left(1-E_{i}\right) \alpha_{i}+E_{i} \tan ^{-1}\left(B_{i} \alpha_{i}\right)\right)\right)
$$

where $i=\{r, f\}$ means rear and front of the vehicle. $D_{i}$, $C_{i}, B_{i}$ and $E_{i}$ are the characteristic coefficients of the tires depending on the road and vehicle operational conditions. $\alpha_{f}$ and $\alpha_{r}$ are respectively the front and rear sideslip angles of tires:

$$
\left\{\begin{array}{l}
\alpha_{f}=\delta_{f}-\beta-\tan ^{-1}\left(\frac{l_{f}}{v_{x}} \dot{\psi} \cos (\beta)\right) \\
\alpha_{r}=-\beta+\tan ^{-1}\left(\frac{l_{r}}{v_{x}} \dot{\psi} \cos (\beta)\right)
\end{array}\right.
$$

For small variations of the sideslip angle $\beta \approx \frac{v_{y}}{v_{x}}$, Equation (4) may be simplified as follows

$$
\left\{\begin{array}{l}
\alpha_{f}=\delta_{f}-\frac{v_{y}}{v_{x}}-\frac{l_{f}}{v_{x}} \dot{\psi} \\
\alpha_{r}=-\frac{v_{y}}{v_{x}}+\frac{l_{r}}{v_{x}} \dot{\psi}
\end{array}\right.
$$

In order to study the behavior of the tire during transient conditions, a typical dynamic model can be used to describe lateral tire force dynamics $F_{y f}$ and $F_{y r}$. In this model presented in [17], lateral forces can be written as:

$$
\left\{\begin{array}{l}
\dot{F}_{y f}=-\frac{v_{x}}{r_{f}} F_{y f}+\frac{v_{x}}{r_{f}} F_{y f}^{S} \\
\dot{F}_{y r}=-\frac{v_{x}}{r_{r}} F_{y r}+\frac{v_{x}}{r_{r}} F_{y r}^{S}
\end{array}\right.
$$

where $r_{f}$ and $r_{r}$ are the relaxation lengths, i.e. approximate distances needed to increase tire forces. $F_{y f}^{S}$ and $F_{y r}^{S}$ are the front and rear steady-state lateral tire forces given by the quasi-static model (2). In normal driving situations, these forces can be assumed to be proportional to sideslip angles $\alpha_{f}$ and $\alpha_{r}$ as follows:

$$
F_{y f}^{S}=c_{f} \alpha_{f}, \quad F_{y r}^{S}=c_{r} \alpha_{r}
$$

where $c_{f}$ and $c_{r}$ represent the cornering stiffness of front and rear tires, respectively, and, can be calculated as $C=$ $B_{i} C D_{i}$, for $i=\{r, f\}$.
Now, the dynamics equations (1) describing lateral and yaw vehicle motions are used in conjunction with the first order transient tire model (5):

$$
\left\{\begin{array}{l}
\dot{v}_{y}=\frac{1}{m}\left(F_{y f}+F_{y r}\right)-v_{x} \dot{\psi} \\
\ddot{\psi}=\frac{1}{I_{z}}\left(l_{f} F_{y f}-l_{r} F_{y r}\right) \\
\dot{F}_{y f}=-\frac{v_{x}}{r_{f}} F_{y f}+\frac{v_{x}}{r_{f}} F_{y f}^{S} \\
\dot{F}_{y r}=-\frac{v_{x}}{r_{r}} F_{y r}+\frac{v_{x}}{r_{r}} F_{y r}^{S}
\end{array}\right.
$$

For design purpose, (7) is transformed using the following change of coordinates:

$$
\left\{\begin{array}{l}
x_{1}(t)=v_{y} \\
x_{2}(t)=\dot{\psi} \\
x_{3}(t)=\frac{1}{m}\left(F_{y f}+F_{y r}\right) \\
x_{4}(t)=\frac{1}{I_{z}}\left(l_{f} F_{y f}-l_{r} F_{y r}\right)
\end{array}\right.
$$

The aim of this transformation is to reduce complexity and dimensionality of the model (7). After some calculation, (7) is rewritten in the following state-space representation:

$$
\begin{aligned}
& \dot{x}(t)=A\left(v_{x}\right) x(t)+B\left(v_{x}\right) u(t) \\
& y(t)=C x(t)
\end{aligned}
$$

where $u(t)=\delta_{f}$ and

$$
\begin{gathered}
A\left(v_{x}\right)=\left[\begin{array}{cccc}
0 & -v_{x} & 1 & 0 \\
0 & 0 & 0 & 1 \\
-\frac{1}{m r}\left(c_{r}+c_{f}\right) & \frac{1}{m_{r}}\left(c_{r} l_{r}-c_{f} l_{f}\right) & -\frac{v_{x}}{r} & 0 \\
\frac{1}{I_{z} r}\left(c_{r} l_{r}-c_{f} l_{f}\right) & -\frac{1}{I_{z} r}\left(c_{r} l_{r}^{2}+c_{f} l_{f}^{2}\right) & 0 & -\frac{v_{x}}{r}
\end{array}\right] \\
B\left(v_{x}\right)=\left[\begin{array}{llll}
0 & 0 & \frac{c_{f}}{m r} v_{x} & \frac{c_{f} l_{f}}{I_{z} r} v_{x}
\end{array}\right]^{T}, \quad C=\left[\begin{array}{cccc}
0 & 1 & 0 & 0 \\
0 & 0 & 1 & 0
\end{array}\right]
\end{gathered}
$$

Here, the available measurements include the yaw rate $\dot{\psi}$ and lateral acceleration $a_{y}=\frac{1}{m}\left(F_{y f}+F_{y r}\right)$. It should be noticed that the cornering stiffnesses $c_{f}$ and $c_{r}$ are the critical uncertain parameters that affect the performance of the vehicle lateral dynamics (9). In this work, it is assumed that the cornering stiffnesses are expressed as a known constant (with $c_{i_{0}}, i \in\{f, r\}$ ) and an uncertainty term $\Delta c_{i}$, $i \in\{f, r\}$ assumed to be unknown but bounded with a priori known bounds:

$$
c_{i}=c_{i_{0}}+\Delta c_{i}, \quad \Delta c_{i} \in\left[\underline{\Delta c_{i}}, \overline{\Delta c}_{i}\right]
$$

It should be also underlined that the longitudinal velocity in (9) is time-varying, which makes obviously, the dynamics of the vehicle, Linear Time-Varying (LPV). To take into account this variation, we propose to represent vehicle dynamics using linear switched systems as an alternative to LPV models. A switched uncertain system where each subsystem operates around a given constant longitudinal velocity value (for example, three subsystems defined for low, average and high longitudinal speed) is adopted. The vehicle model (9) can then be represented by a switched uncertain linear system as follows:

$$
\left\{\begin{array}{l}
\dot{x}(t)=\left(A_{\sigma(t)}+\Delta A_{\sigma(t)}(t)\right) x(t)+\left(B_{\sigma(t)}+\Delta B_{\sigma(t)}(t)\right) u(t) \\
y(t)=C x(t)
\end{array}\right.
$$

where $x(t) \in \mathbb{R}^{n}$ is the state, $u(t) \in \mathbb{R}^{m}$ is the control input, $y(t) \in \mathbb{R}^{p}$ is the measured output. $\sigma(t): \mathbb{R}^{+} \rightarrow I=$ $\{1,2, \ldots, N\}$ is the switching signal, assumed to be available 
in real time and depends on the vehicle longitudinal velocity measurement. $N$ denotes the number of subsystems. $A_{\sigma(t)} \in$ $\mathbb{R}^{n \times n}$ and $B_{\sigma(t)} \in \mathbb{R}^{n \times m}$ are known constant matrices. $\Delta A_{\sigma(t)} \in \mathbb{R}^{n \times n}$ and $\Delta B_{\sigma(t)} \in \mathbb{R}^{n \times m}$ are unknown but bounded matrices. These matrices are given in (12) and (13). For easiness of development, system (11) is rewritten as:

$$
\left\{\begin{array}{l}
\dot{x}(t)=A_{\sigma(t)} x(t)+B_{\sigma(t)} u(t)+\omega_{\sigma(t)}(t) \\
y(t)=C x(t)
\end{array}\right.
$$

where $\omega_{\sigma(t)}(t)=\Delta A_{\sigma(t)}(t) x(t)+\Delta B_{\sigma(t)}(t) u(t)$. Furthermore, the steering angle input $u=\delta_{f}$ is supposed to belong to an admissible set of inputs as

$$
\underline{u}(t) \leq u(t) \leq \bar{u}(t)
$$

The initial condition at the instant $t_{0}, x\left(t_{0}\right) \in \mathbb{R}^{n}$ is assumed to be bounded by two known bounds $\underline{x}\left(t_{0}\right)$ and $\bar{x}\left(t_{0}\right)$ :

$$
\underline{x}\left(t_{0}\right) \leq x\left(t_{0}\right) \leq \bar{x}\left(t_{0}\right)
$$

The additive disturbances $\omega_{\sigma(t)}(t) \in \mathbb{R}^{n}$ in (14) is assumed to be unknown but upper and lower bounded ${ }^{1}$ as follows:

$$
-\infty \leq \underline{\omega}_{\sigma(t)}(t) \leq \omega_{\sigma(t)}(t) \leq \bar{\omega}_{\sigma(t)}(t) \leq+\infty
$$

where $\underline{\omega}_{\sigma(t)}(t)$ and $\bar{\omega}_{\sigma(t)}(t)$ are two a priori known functions $\forall \sigma(t)$. Since the state space matrices $\Delta A_{\sigma(t)}$ and $\Delta B_{\sigma(t)}$ in (13) depend on $\Delta c_{f}$ and $\Delta c_{r}$ affinely, then using (10), we obtain the following inequalities:

$$
\begin{aligned}
& \underline{\Delta A}_{\sigma(t)} \leq \Delta A_{\sigma(t)} \leq \overline{\Delta A}_{\sigma(t)} \\
& \underline{\Delta B}_{\sigma(t)} \leq \Delta B_{\sigma(t)} \leq \overline{\Delta B}_{\sigma(t)}
\end{aligned}
$$

\section{SWITCHED INTERVAL OBSERVER DESIGN}

In this section we give some preliminary definitions and lemmas related to interval observer design. Then, the switched interval observer structure is presented. The conditions for the existence of such an observer are given. By combining Input-to-State Stability (ISS) concept [21] with Common Lyapunov functions [7], the derived LMI-based optimization problem guarantees an ultimate bound on the interval estimation error.

\section{A. Preliminaries}

Definition $1 A_{\sigma(t)}$ is said to be a Metzler matrix if all its off-diagonal entries are non-negative for any $\sigma(t) \in I$.

Lemma 1 ( [16]) A matrix $A$ is Metzler if and only if there exists a constant $\eta \in \mathbb{R}_{+}$such that $A+\eta I_{n} \geq 0$, where $I_{n}$ is an $n \times n$ identity matrix and $\geq$ is interpreted element-wise.

Lemma 2 ( [1]) The switched system

$$
\dot{x}(t)=A_{\sigma(t)} x(t)+\omega_{\sigma(t)}(t), \quad x\left(t_{0}\right)=0
$$

is positive, i.e., $x(t) \geq 0$, if and only if $A_{\sigma(t)}$ is a Metzler matrix, $\omega_{\sigma(t)} \geq 0$ and $x\left(t_{0}\right) \geq 0$, for any $\sigma(t) \in I$.

Lemma 3 ( [2]) Let the vector $x \in \mathbb{R}^{n}$ be a variable vector with given bounds $\bar{x}, \underline{x} \in \mathbb{R}^{n}$ such that $\underline{x} \leq x \leq \bar{x}$.

${ }^{1}$ Since, in the context of vehicle application, the state $x(t)$ and input $u(t)$ are physically bounded.
1) If $A \in \mathbb{R}^{n \times n}$ is a constant matrix, then

$$
A^{+} \underline{x}-A^{-} \bar{x} \leq A x \leq A^{+} \bar{x}-A^{-} \underline{x} .
$$

2) If $A \in \mathbb{R}^{n_{x} \times n_{x}}$ is a variable matrix such that $\underline{A} \leq$ $A \leq \bar{A}$ for some $\underline{A}, \bar{A} \in \mathbb{R}^{n \times n}$, then

$$
\begin{aligned}
& \underline{A}^{+} \underline{x}^{+}-\bar{A}^{+} \underline{x}^{-}-\underline{A}^{-} \bar{x}^{+}+\bar{A}^{-} \bar{x}^{-} \leq A x \\
& \leq \bar{A}^{+} \bar{x}^{+}-\bar{A}^{-} \underline{x}^{+}-\underline{A}^{+} \bar{x}^{-}+\underline{A}^{-} \underline{x}^{-}
\end{aligned} .
$$

where $\star^{+}=\max (0, \star)$ and $\star^{-}=\star^{+}-\star$.

\section{B. Switched Interval Observer structure}

In this paper, a new interval observer structure is proposed for the uncertain switched linear systems (14). The aim is to calculate an interval of admissible values of the state vector $x(t)$, i.e. $x(t) \in[\underline{x}(t), \bar{x}(t)]$, where $\underline{x}(t)$ and $\bar{x}(t)$ are two bounds fulfilling the following property:

$$
\underline{x}(t) \leq x(t) \leq \bar{x}(t), \quad \forall t \geq t_{0}
$$

To this end, a switched interval observer can be designed for (14) as:

$$
\left\{\begin{array}{l}
\dot{\bar{\xi}}(t)=N_{\sigma(t)} \bar{\xi}(t)+J_{\sigma(t)} y(t)+H_{\sigma(t)} u(t) \\
+\bar{\Omega}_{\sigma(t)}(t) \\
\bar{\xi}\left(t_{0}\right)=\bar{x}\left(t_{0}\right)-E_{\sigma\left(t_{0}\right)} y\left(t_{0}\right) \\
\bar{x}(t)=\bar{\xi}(t)+E_{\sigma(t)} y(t) \\
\dot{\xi}(t)=N_{\sigma(t)} \underline{\xi}(t)+J_{\sigma(t)} y(t)+H_{\sigma(t)} u(t) \\
\underline{+} \underline{\Omega}_{\sigma(t)}(t) \\
\underline{\xi}\left(t_{0}\right)=\underline{x}\left(t_{0}\right)-E_{\sigma\left(t_{0}\right)} y\left(t_{0}\right) \\
\underline{x}(t)=\underline{\xi}(t)+E_{\sigma(t)} y(t)
\end{array}\right.
$$

where $\xi(t), \quad \bar{\xi}(t) \in \mathbb{R}^{n}$ are intermediate variables, $\bar{x}(t), \underline{x}(t) \in \mathbb{R}^{n}$ are the estimated upper and lower bounds of $x(t)$, respectively. $\bar{\Omega}_{\sigma(t)}(t), \underline{\Omega}_{\sigma(t)}(t) \in \mathbb{R}^{n}$ are given as

$$
\begin{aligned}
& \bar{\Omega}_{\sigma(t)}(t)=P_{\sigma(t)}^{+} \bar{\omega}_{\sigma(t)}(t)-P_{\sigma(t)}^{-} \underline{\omega}_{\sigma(t)}(t) \\
& \underline{\Omega}_{\sigma(t)}(t)=P_{\sigma(t)}^{+} \underline{\omega}_{\sigma(t)}(t)-P_{\sigma(t)}^{-} \bar{\omega}_{\sigma(t)}(t)
\end{aligned}
$$

$\bar{\omega}_{\sigma(t)}(t)$ and $\underline{\omega}_{\sigma(t)}(t)$ are directly obtained by applying Lemma 3 and using (22) and (15):

$$
\begin{aligned}
& \bar{\omega}_{\sigma(t)}(t)=\overline{\Delta A}_{\sigma(t)}^{+} \bar{x}^{+}(t)-\underline{\Delta A}_{\sigma(t)}^{+} \bar{x}^{-}(t) \\
& -\overline{\Delta A}_{\sigma(t)}^{-} \underline{x}^{+}(t)+\underline{\Delta A}_{\sigma(t)}^{-} \underline{x}^{-}(t)+\overline{\Delta B}_{\sigma(t)}^{+} \bar{u}^{+}(t) \\
& -\underline{\Delta B}_{\sigma(t)}^{+} \bar{u}^{-}(t)-\overline{\Delta B}_{\sigma(t)}^{-} \underline{u}^{+}(t)+\underline{\Delta B}_{\sigma(t)}^{-} \underline{u}^{-}(t) \\
& \underline{\omega}_{\sigma(t)}(t)=\Delta A_{\sigma(t)}^{+} \underline{x}^{+}(t)-\overline{\Delta A}_{\sigma(t)}^{+} \underline{x}^{-}(t) \\
& -\underline{\Delta A}_{\sigma(t)}^{-} \bar{x}^{+}(t)+\overline{\Delta A}_{\sigma(t)}^{-} \bar{x}^{-}(t)+\Delta B_{\sigma(t)}^{+} \underline{u}^{+}(t) \\
& -\overline{\Delta B}_{\sigma(t)}^{+} \underline{u}^{-}(t)-\underline{\Delta B}
\end{aligned}
$$

In (23), $N_{\sigma(t)}, J_{\sigma(t)}, H_{\sigma(t)}, E_{\sigma(t)}$ and $P_{\sigma(t)}(t)$ are switched constant matrices to be designed such that (22) holds $\forall t \geq t_{0}$.

\section{LMI formulation}

By defining the upper and lower estimation errors as

$$
\begin{aligned}
& \bar{e}(t)=\bar{x}(t)-x(t) \\
& \underline{e}(t)=x(t)-\underline{x}(t)
\end{aligned}
$$

the inequality (22) is equivalent to show that the upper and lower estimation errors (26) are positive for all initial 


$$
\begin{aligned}
& A_{\sigma(t)}=\left[\begin{array}{cccc}
0 & -v_{x}^{\sigma(t)} & 1 & 0 \\
0 & 0 & 0 & 1 \\
-\frac{1}{m r}\left(c_{r_{0}}+c_{l_{0}}\right) & \frac{1}{m r}\left(c_{r_{0}} l_{r}-c_{f_{0}} l_{f}\right) & -\frac{v_{x}^{\sigma(t)}}{r} & 0 \\
\frac{1}{I_{z} r}\left(c_{r_{0}} l_{r}-c_{f_{0}} l_{f}\right) & -\frac{1}{I_{z} r}\left(c_{r_{0}} l_{r}^{2}+c_{f_{0}} l_{f}^{2}\right) & 0 & -\frac{v_{x}^{\sigma(t)}}{r}
\end{array}\right], \quad B_{\sigma(t)}=\left[\begin{array}{c}
0 \\
0 \\
\frac{c_{f_{0}}}{m r_{0}} v_{x}^{\sigma(t)} \\
\frac{c_{f_{0}} f_{f}}{I_{z} r} v_{x}^{\sigma(t)}
\end{array}\right] \\
& \Delta A_{\sigma(t)}=\left[\begin{array}{cccc}
0 & 0 & 0 & 0 \\
0 & 0 & 0 & 0 \\
-\frac{1}{m r}\left(\Delta c_{r}+\Delta c_{f}\right) & \frac{1}{m r}\left(\Delta c_{r} l_{r}-\Delta c_{f} l_{f}\right) & 0 & 0 \\
\frac{1}{I_{z} r}\left(\Delta c_{r} l_{r}-\Delta c_{f} l_{f}\right) & -\frac{1}{I_{z} r}\left(\Delta c_{r} l_{r}^{2}+\Delta c_{f} l_{f}^{2}\right) & 0 & 0
\end{array}\right], \quad \Delta B_{\sigma(t)}=\left[\begin{array}{c}
0 \\
0 \\
\frac{\Delta c_{f}}{m r^{2}} v_{x}^{\sigma(t)} \\
\frac{\Delta c_{f} l_{f}}{I_{z} r} v_{x}^{\sigma(t)}
\end{array}\right]
\end{aligned}
$$

conditions $\underline{e}\left(t_{0}\right)=x\left(t_{0}\right)-\underline{x}\left(t_{0}\right)$ and $\bar{e}\left(t_{0}\right)=\bar{x}\left(t_{0}\right)-x\left(t_{0}\right)$. Using (14) and (23), we obtain

$$
\begin{aligned}
& \bar{e}(t)=\bar{\xi}(t)+E_{\sigma(t)} y(t)-x(t) \\
& \underline{e}(t)=x(t)-\underline{\xi}(t)-E_{\sigma(t)} y(t)
\end{aligned}
$$

it follows that

$$
\begin{aligned}
& \bar{e}(t)=\bar{\xi}(t)-\left(I_{n}-E_{\sigma(t)} C_{\sigma(t)}\right) x(t) \\
& \underline{e}(t)=\left(I_{n}-E_{\sigma(t)} C_{\sigma(t)}\right) x(t)-\underline{\xi}(t)
\end{aligned}
$$

Let us set $P_{\sigma(t)}=I_{n}-E_{\sigma(t)} C_{\sigma(t)}$, the upper and lower errors dynamics are given by

$$
\begin{aligned}
& \dot{\bar{e}}(t)=N_{\sigma(t)} \bar{e}(t)+\left(H_{\sigma(t)}-P_{\sigma(t)} B_{\sigma(t)}\right) u(t) \\
& -\left(P_{\sigma(t)} A_{\sigma(t)}-N_{\sigma(t)} P_{\sigma(t)}-J_{\sigma(t)} C_{\sigma(t)}\right) x(t)+ \\
& \bar{\Omega}_{\sigma(t)}(t)-P_{\sigma(t)} \omega_{\sigma(t)}(t) \\
& \dot{e}(t)=N_{\sigma(t)} \underline{e}(t)-\left(H_{\sigma(t)}-P_{\sigma(t)} B_{\sigma(t)}\right) u(t) \\
& +\left(P_{\sigma(t)} A_{\sigma(t)}-N_{\sigma(t)} P_{\sigma(t)}-J_{\sigma(t)} C_{\sigma(t)}\right) x(t)+ \\
& P_{\sigma(t)} \omega_{\sigma(t)}(t)-\underline{\Omega}_{\sigma(t)}(t)
\end{aligned}
$$

Assuming that matrices are chosen such that the following constraints hold:

$$
\begin{gathered}
P_{\sigma(t)} A_{\sigma(t)}-N_{\sigma(t)} P_{\sigma(t)}-J_{\sigma(t)} C_{\sigma(t)}=0 \\
H_{\sigma(t)}-P_{\sigma(t)} B_{\sigma(t)}=0
\end{gathered}
$$

then, (29) becomes

$$
\begin{aligned}
& \dot{\bar{e}}(t)=N_{\sigma(t)} \bar{e}(t)+\bar{\Omega}_{\sigma(t)}(t)-P_{\sigma(t)} \omega_{\sigma(t)}(t) \\
& \underline{\dot{e}}(t)=N_{\sigma(t)} \underline{e}(t)+P_{\sigma(t)} \omega_{\sigma(t)}(t)-\underline{\Omega}_{\sigma(t)}(t)
\end{aligned}
$$

According to Lemma 3, for two given functions $\bar{\omega}_{\sigma(t)}(t)$ and $\underline{\omega}_{\sigma(t)}(t)$ such that $\underline{\omega}_{\sigma(t)}(t) \leq \omega_{\sigma(t)}(t) \leq \bar{\omega}_{\sigma(t)}(t)$, it is clear that $\bar{\Omega}_{\sigma(t)}(t)-P_{\sigma(t)} \omega_{\sigma(t)}(t)$ and $P_{\sigma(t)} \omega_{\sigma(t)}(t)-\underline{\Omega}_{\sigma(t)}(t)$ are nonnegative $\forall \sigma(t)$ and $t \geq t_{0}$. From (31) and based on Lemma 2, it follows that (22) is satisfied, provided that $N_{\sigma(t)}$ is a Metzler matrix and $\underline{x}\left(t_{0}\right) \leq x\left(t_{0}\right) \leq \bar{x}\left(t_{0}\right)$. However, only designing a Metzler matrix $N_{\sigma(t)}$ is not sufficient to obtain an accurate interval estimation.

It should be noticed that, for achieving better performance, the lower and upper estimated bounds should be as close as possible to $x(t)$. To this end, let us consider the interval error $e(t)=\bar{x}(t)-\underline{x}(t)=\bar{e}(t)+\underline{e}(t)$ governed by the following differential equation:

$$
\dot{e}(t)=N_{\sigma(t)} e(t)+\Omega_{\sigma(t)}(t)
$$

where $\Omega_{\sigma(t)}(t)=\bar{\Omega}_{\sigma(t)}(t)-\underline{\Omega}_{\sigma(t)}(t)$. Therefore, and, in order to use the well known results obtained for the classical full order state observer without unknown inputs, equation (30a) is written as

$$
N_{\sigma(t)}=P_{\sigma(t)} A_{\sigma(t)}-K_{\sigma(t)} C_{\sigma(t)}
$$

where $K_{\sigma(t)}=J_{\sigma(t)}-N_{\sigma(t)} E_{\sigma(t)}$. Theorem 1 which is our main contribution gives sufficient LMIs conditions that ensure the following specifications

- $\bar{e}(t) \geq 0$, i.e. $\bar{x}(t) \geq x(t)$, and, $\underline{e}(t) \geq 0$, i.e. $x(t) \geq$ $\underline{x}(t)$

- $e(t)$ approaches zero asymptotically, i.e. $\bar{x}(t) \rightarrow \underline{x}(t) \rightarrow$ $x(t)$, when $\Omega_{\sigma(t)}(t)=0$;

- Bounded and tight interval error, when $\Omega_{\sigma(t)}(t) \neq 0$.

Theorem 1 If there exist a positive diagonal matrix $Q$, matrices $W_{i}$ and $X_{i}, \beta>\alpha>0, \gamma>0$ for a given $\eta \geq 0$, $\varepsilon>0$, such that for all $i \in I$,

$$
\begin{gathered}
\min _{Q, W_{i}, X_{i}} \gamma \\
\alpha I_{n} \preceq Q \preceq \beta I_{n} \\
{\left[\begin{array}{cc}
A_{i}{ }^{T} Q-A_{i}{ }^{T} C^{T} X_{i}^{T}-C^{T} W_{i}^{T}+ & \\
Q A_{i}-X_{i} C A_{i}-W_{i} C+\varepsilon Q & Q \\
Q & -\gamma I_{n}
\end{array}\right] \prec 0} \\
Q A_{i}-X_{i} C A_{i}-W_{i} C+\eta Q \geq 0
\end{gathered}
$$

hold, then the system (23) can estimate the lower and upper bounds of the state vector $x(t)$ with $E_{i}=Q^{-1} X_{i}$ and $K_{i}=$ $Q^{-1} W_{i}$.

Furthermore, the interval error (32) is Input-to-State Stable with respect to $\Omega_{i}(t), \forall i \in I$ for arbitrary switching signal, then, if $\sup \left\|\Omega_{i}(t)\right\| \leq \Omega_{\max }, \forall i \in I$, the error satisfies

$$
\lim _{t \rightarrow \infty}\|e(t)\|_{2} \leq \sqrt{\frac{\gamma}{\alpha \varepsilon}} \max _{i \in I}\left\|\Omega_{i}(t)\right\|_{\infty}
$$

Proof. For the switched system (32), let us choose the following common Quadratic ISS-Lyapunov function

$$
V(e(t))=e^{T}(t) Q e(t)
$$

where $Q$ is a positive diagonal matrix. Taking the time derivative of the Lyapunov function $V(e(t))$ along the solution of the interval error dynamics (29), we have

$$
\begin{aligned}
& \dot{V}(e(t))=e^{T}(t)\left(N_{\sigma(t)}^{T} Q+Q N_{\sigma(t)}\right) e(t)+ \\
& \Omega_{\sigma(t)}^{T}(t) Q e(t)+e^{T}(t) Q \Omega_{\sigma(t)}(t)
\end{aligned}
$$

By adding and subtracting the terms $\varepsilon e^{T}(t) Q e(t)$ and $-\gamma \Omega_{\sigma(t)}^{T}(t) \Omega_{\sigma(t)}(t)$, (39) becomes

$$
\begin{aligned}
& \dot{V}(e(t))=\left[\begin{array}{ll}
e^{T}(t) & \Omega_{\sigma(t)}^{T}(t)
\end{array}\right]^{T} \Lambda_{\sigma(t)}\left[\begin{array}{ll}
e(t) & \Omega_{\sigma(t)}(t)
\end{array}\right]^{T} \\
& -\varepsilon V(e(t))+\gamma \Omega_{\sigma(t)}^{T}(t) \Omega_{\sigma(t)}(t)
\end{aligned}
$$

where

$$
\Lambda_{\sigma(t)}=\left[\begin{array}{cc}
N_{\sigma(t)}^{T} Q+Q N_{\sigma(t)}+\varepsilon Q & Q \\
Q & -\gamma I_{n}
\end{array}\right]
$$


replacing $N_{\sigma(t)}$ by (33) and denote $X_{\sigma(t)}=Q E_{\sigma(t)}$, $W_{\sigma(t)}=Q K_{\sigma(t)}, \Lambda_{\sigma(t)}$ is rewritten as follows

$$
\Lambda_{\sigma(t)}=\left[\begin{array}{cc}
A_{\sigma(t)}^{T} Q-A_{\sigma(t)}^{T} C^{T} X_{\sigma(t)}^{T}-C^{T} W_{\sigma(t)}^{T}+ & \\
Q A_{\sigma(t)}-X_{\sigma(t)} C A_{\sigma(t)}-W_{\sigma(t)} C+\varepsilon Q & Q \\
Q & -\gamma I_{n}
\end{array}\right]
$$

satisfying (35), $\forall i \in I$, leads to

$$
\dot{V}(e(t))<-\varepsilon V(e(t))+\gamma \Omega_{\sigma(t)}^{T}(t) \Omega_{\sigma(t)}(t)
$$

integrating (41) over the interval $\left[t_{k}, t\right)$ implies that

$$
V(e(t))<\mathrm{e}^{-\varepsilon\left(t-t_{k}\right)} V\left(e\left(t_{k}\right)\right)+\gamma \int_{t_{k}}^{t} \mathrm{e}^{-\varepsilon\left(\left(t-t_{k}\right)-s\right)}\left\|\Omega_{\sigma(t)}(s)\right\|_{2}^{2} \mathrm{~d} s
$$

Using (34), we obtain

$$
\begin{aligned}
& \text { Using (34), we obtain } \\
& \|e(t)\|_{2}<\frac{1}{\sqrt{\alpha}}\left(\mathrm{e}^{-\varepsilon\left(t-t_{k}\right)} V\left(e\left(t_{k}\right)\right)+\frac{\gamma}{\varepsilon}\left\|\Omega_{\sigma(t)}(t)\right\|_{\infty}^{2}\right)^{\frac{1}{2}}
\end{aligned}
$$

Hence, when $t \rightarrow \infty$ the exponential converges towards zero, implies that:

$$
\lim _{t \rightarrow \infty}\|e(t)\|_{2} \leq \sqrt{\frac{\gamma}{\alpha \varepsilon}} \max _{i \in I}\left\|\Omega_{i}(t)\right\|_{\infty}
$$

then, (37) is directly deduced and the ISS property is proven. The radius of the convergence region $\mathcal{D}$ is upper bounded by $\sqrt{\frac{\gamma}{\alpha \varepsilon}} \max _{i \in I}\left\|\Omega_{i}(t)\right\|_{\infty}$. By minimizing $\gamma$, the convergence set $\mathcal{D}$ is made as small as possible, thus, the width of the estimated interval is narrowed. On the other hand, according to Lemma 1, $N_{\sigma(t)}$ defined in (33) is Metzler if

$$
N_{i}+\eta I_{n} \geq 0, \forall i \in I
$$

multiplying in the left side by $Q$ and using (33) together with the change of coordinates $X_{i}=Q E_{i}, W_{i}=Q K_{i}$, (36) is obtained.

Remark 1 Note that the existence of the Common quadratic ISS-Lyapunov function (38) ensures that the interval observer (23) is Uniformly Input-to-state Stable. This issue is usually used when there is no restriction on the switching signals and requires that all the subsystems are ISS-stable. Then the stability of the switched system is insured under arbitrary switching [20]. The word "Uniform" refers to uniformity with respect to switching signals. We refer the reader to [8] for some related results on common Lyapunov functions existence problem not covered here.

\section{Simulation RESUlts USING REAL DATA}

Test of the proposed vehicle lateral dynamics estimator are carried out in Matlab/Simulink. The performance of the proposed design was tested using real data recorded previously with a prototype vehicle. The front input steering angle, $\delta_{f}$, is measured using an absolute encoder. The longitudinal velocity, $v_{x}$, is measured using an odometer. Yaw rate and lateral acceleration are obtained using a three-axis inertial unit. All these data profiles are presented in Figure 1. Lateral velocity appearing in Figure 3 is measured using a Correvit sensor and used only for validation.

The simulation is conducted in accordance with the following switching signal (See Figure 2) using the measured longitudinal velocity $v_{x}$ :

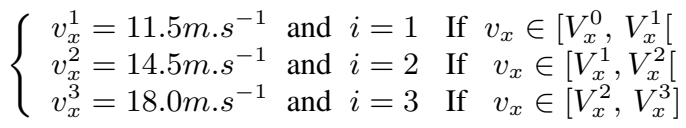

where $V_{x}^{0}=10 \mathrm{~m} . \mathrm{s}^{-1}, V_{x}^{1}=13 \mathrm{~m} . \mathrm{s}^{-1}$ and $V_{x}^{2}=16 \mathrm{~m} . \mathrm{s}^{-1}$ and $V_{x}^{3}=20 \mathrm{~m} . \mathrm{s}^{-1}$. Three local models are obtained, i.e., $N=3$ and $i=1,2,3$. The stability and cooperativity of the proposed interval observer (23) are guaranteed by solving the set of LMIs in Theorem 1. By choosing $\varepsilon=0.5$ and $\eta=7$. The obtained matrices are omitted for brevity.

After estimating the states of the switched system (9) in the new coordinates (8), the estimated lateral forces can be obtained easily from the following algebraic equations:

$$
\begin{array}{ll}
\bar{F}_{y f}=\frac{m l_{r} \bar{x}_{3}(t)+I_{z} \bar{x}_{4}(t)}{l f+l r}, & \underline{F}_{y f}=\frac{m l_{r} \underline{x}_{3}(t)+I_{z} \underline{x}_{4}(t)}{l f+l r} \\
\bar{F}_{y r}=\frac{m l_{f} \bar{x}_{3}(t)-I_{z} \underline{x}_{4}(t)}{l f+l r}, & \underline{F}_{y r}=\frac{m l_{f} \underline{\underline{x}}_{3}(t)-I_{z} \bar{x}_{4}(t)}{l f+l r}
\end{array}
$$

Interval estimation results are shown in Figure 3. One can observe that the switched interval observer (23) provides satisfactory upper and lower estimations of the lateral velocity and yaw rate measurements $v_{y}^{m}$ and $\dot{\psi}^{m}$. Since we do not possess the real measurement of the lateral forces to validate our results, we will take advantage of the lateral acceleration measure, therefore, knowing that

$$
m a_{y}=F_{y f}+F_{y r}
$$

where $m$ is the vehicle mass, then, the following physical constraints should be satisfied

$$
\underline{F}_{y f}+\underline{F}_{y r} \leq m a_{y} \leq \bar{F}_{y f}+\bar{F}_{y r}
$$

The Figure 4 shows the values of the product $m a_{y}$ plotted with the upper and lower bounds in (47). Remark that the resulting interval is accurate and enclose the real trajectory which validates the proposed lateral tire-road forces interval estimation.
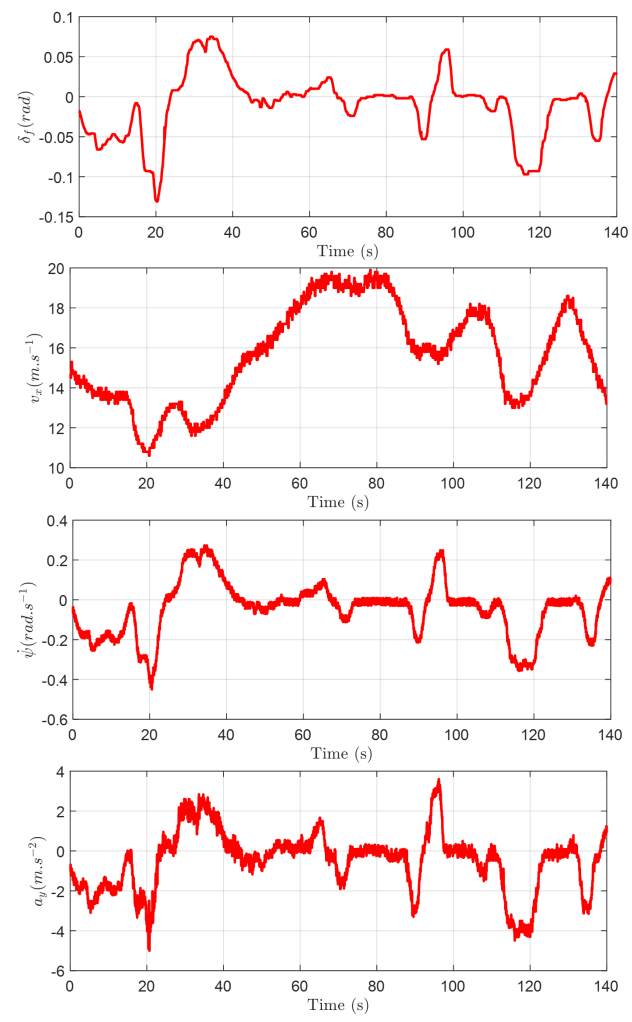

Fig. 1. Steering angle $\delta_{f}$, Longitudinal velocity $v_{x}$, Yaw rate $\dot{\psi}$ and Lateral acceleration $a_{y}$. 


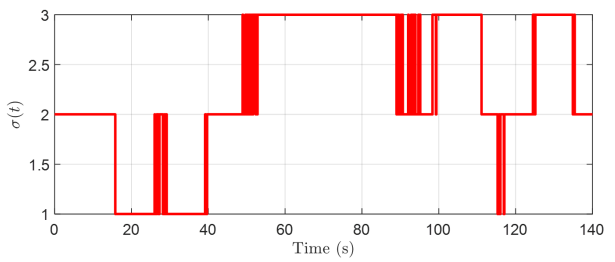

Fig. 2. Switching law $\sigma(t)$.
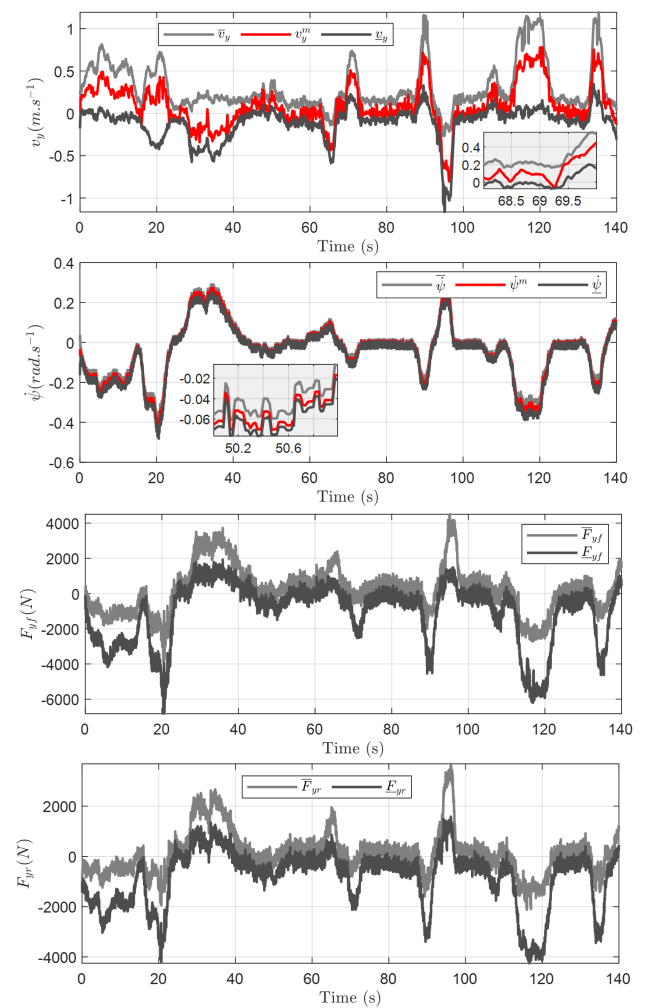

Fig. 3. Interval estimation of vehicle lateral velocity $v_{y}$, yaw rate $\dot{\psi}$, front and rear lateral tire force $F_{y f}$ and $F_{y r}$.

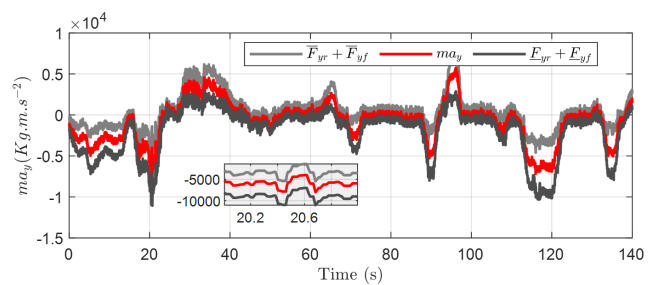

Fig. 4. Interval estimation of the signal $m a_{y}$.

\section{CONCLusion}

In this paper, an interval estimation procedure for vehicle lateral velocity and lateral tire forces, two of the most important parameters affecting vehicle stability, is proposed. The switched interval observer design based on ISS concept and common Lyapunov theory has been presented. The existence conditions have been established in terms of LMIs. A comparison with real experimental data demonstrates the potential of the presented interval estimation process. For future investigations, it is interesting to extend the results for application in fault diagnosis and fault tolerant control for practical implementation.

\section{REFERENCES}

[1] Franco Blanchini, Patrizio Colaneri, Maria Elena Valcher, et al. Switched positive linear systems. Foundations and Trends $\AA$ in Systems and Control, 2(2):101-273, 2015.

[2] Denis Efimov, Leonid Fridman, Tarek Raissi, Ali Zolghadri, and Ramatou Seydou. Interval estimation for lpv systems applying high order sliding mode techniques. Automatica, 48(9):2365-2371, 2012.

[3] Nicolas Ellero, David Gucik-Derigny, and David Henry. Unknown input interval observer with $h_{\infty}$ and d-stability performance. IFAC Proceedings Volumes, 50(1):6251 - 6258, 2017.

[4] Haifa Ethabet, Djahid Rabehi, Denis Efimov, and Tarek Raïssi. Interval estimation for continuous-time switched linear systems. Automatica, 90:230-238, 2018

[5] Jean-Luc Gouzé, Alain Rapaport, and Mohamed Zakaria Hadj-Sadok. Interval observers for uncertain biological systems. Ecological modelling, 133(1):45-56, 2000.

[6] Sara Ifqir, Vicenç Puig, Dalil Ichalal, Naima Ait-Oufroukh, and Saïd Mammar. Robust fault detection for vehicle lateral dynamics: A zonotope-based set-membership approach. The 21st International Conference on Intelligent Transportation Systems (ITSC), 2018.

[7] Daniel Liberzon. Switching in systems and control. Springer Science \& Business Media, 2012.

[8] Daniel Liberzon and A Stephen Morse. Basic problems in stability and design of switched systems. IEEE control systems magazine, 19(5):59-70, 1999.

[9] Anders Lie, Claes Tingvall, Maria Krafft, and Anders Kullgren. The effectiveness of esp (electronic stability program) in reducing real life accidents. Traffic Injury Prevention, 5(1):37-41, 2004.

[10] Sard Mammar, Sébastien Glaser, and Mariana Netto. Vehicle lateral dynamics estimation using unknown input proportional-integral observers. In American Control Conference, 2006, pages 6-pp. IEEE, 2006.

[11] Frédéric Mazenc and Olivier Bernard. Asymptotically stable interval observers for planar systems with complex poles. IEEE Transactions on Automatic Control, 55(2):523-527, 2010.

[12] Hans B Pacejka and Egbert Bakker. The magic formula tyre model. Vehicle system dynamics, 21(S1):1-18, 1992.

[13] Jochen Pohl, Wolfgang Birk, and Lena Westervall. A driverdistraction-based lane-keeping assistance system. Proceedings of the Institution of Mechanical Engineers, Part I: Journal of Systems and Control Engineering, 221(4):541-552, 2007.

[14] Tarek Raïssi and Denis Efimov. Some recent results on the design and implementation of interval observers for uncertain systems. atAutomatisierungstechnik, 66(3):213-224, 2018.

[15] Rajesh Rajamani. Vehicle dynamics and control. Springer Science \& Business Media, 2011.

[16] M Ait Rami, Chun Hung Cheng, and C De Prada. Tight robust interval observers: an LP approach. In Decision and Control, 2008. CDC 2008. 47th IEEE Conference on, pages 2967-2972. IEEE, 2008.

[17] Georg Rill. First order tire dynamics. In 3rd European Conference on Computational Mechanics Solids, Structures and Coupled Problems in Engineering CA Mota Soares et. al.(ed.) Lisbon, Portugal, pages 5-8, 2006.

[18] Jihan Ryu and J Christian Gerdes. Estimation of vehicle roll and road bank angle. In American Control Conference, 2004. Proceedings of the 2004, volume 3, pages 2110-2115. IEEE, 2004.

[19] Yazid Sebsadji, Sébastien Glaser, Said Mammar, and Jamil Dakhlallah. Road slope and vehicle dynamics estimation. In American Control Conference, 2008, pages 4603-4608. IEEE, 2008.

[20] RN Shorten and KS Narendra. On the stability and existence of common lyapunov functions for stable linear switching systems. In Decision and Control, 1998. Proceedings of the 37th IEEE Conference on, volume 4, pages 3723-3724. IEEE, 1998.

[21] Eduardo D Sontag and Yuan Wang. Notions of input to output stability. Systems \& Control Letters, 38(4-5):235-248, 1999.

[22] Joanny Stephant, Ali Charara, and Dominique Meizel. Virtual sensor: Application to vehicle sideslip angle and transversal forces. IEEE Transactions on industrial electronics, 51(2):278-289, 2004.

[23] Keisuke Suzuki and Håkan Jansson. An analysis of drivers steering behaviour during auditory or haptic warnings for the designing of lane departure warning system. JSAE review, 24(1):65-70, 2003.

[24] Bart Van Arem, Cornelie JG Van Driel, and Ruben Visser. The impact of cooperative adaptive cruise control on traffic-flow characteristics. IEEE Transactions on intelligent transportation systems, 7(4):429436, 2006. 